\title{
Exercise Behavior, Facilitators and Barriers among Socio-economically Disadvantaged African American Young Adults
}

\author{
Maria Kosma ${ }^{1 *}$, David R. Buchanan ${ }^{2}$ \\ ${ }^{I}$ School of Kinesiology, Louisiana State University 331 Peabody Hall, Baton Rouge, Louisiana 70803, Baton Rouge, USA, \\ ${ }^{2}$ School of Public Health \& Health Sciences, University of Massachusetts 306 Arnold House, Amherst 01003, Amherst, MA \\ Corresponding Author: Maria Kosma, E-mail: mkosma@1su.edu
}

\section{ARTICLE INFO}

Article history

Received: December 12, 2017

Accepted: March 15, 2018

Published: April 30, 2018

Volume: 6 Issue: 2

Conflicts of interest: None

Funding: None

\begin{abstract}
Background: Although exercise participation has numerous benefits among young adults, socio-economically disadvantaged ethnic minorities tend to be less active than their White counterparts of higher SES. Instead of relying on logical positivism in exercise promotion, a phronetic (humanistic) approach may better assist with understanding exercise behavior. Objective: The study purpose was to examine the exercise behavior and qualitatively distinct exercise values (e.g., activity and inactivity reasons) among socio-economically disadvantaged African American young adults. Method: This was a phronetic, qualitative study among 14 African American young adults $\left(\mathrm{M}_{\text {age }}=32.97\right.$ years old \pm 14.13$)$, who attended General Educational Development classes in an inner-city learning center. An in-depth and dialogical interview process was conducted regarding exercise behavior, positive and negative exercise experiences, reasons for exercise participation or not, exercise behavior of participants' peers and significant others, and neighborhood safety. Results: Only three men met the minimum aerobic exercise recommendations and their main activity was basketball. Three individuals were somewhat active, while the rest of the participants were inactive. Based on the phronetic, thematic analysis, two themes emerged. Exercise facilitators included enjoyment (from skill and fitness development in a playful setting), health improvement, weight loss and toned physique, and utilitarian purpose (i.e., karate to work for campus security). Exercise barriers included time constraints and other priorities (school, work, caretaking), injuries, accessibility and cost issues, safety issues (unsafe neighborhoods), personality (lack of motivation and self-discipline), and undesirable results on appearance and performance. Conclusion: Exercise promoters should emphasize: a) playful, culturally meaningful, and socially supported activities to increase fitness, skill development, and enjoyment; b) policy change via the provision of parental leave for both parents; c) safe and accessible exercise settings, especially among women of lower SES; d) information about safe exercises and injury recovery; and e) fit and healthy physiques for personal fulfilment and enjoyment.
\end{abstract}

Key words: African Americans, Social Justice, Exercise, Culture, Physical Fitness

\section{INTRODUCTION}

Physical activity has numerous benefits among young adults, including increased cardiorespiratory fitness, psychological health, cognitive capacity, sleep time and quality, and energy levels (Gerber et al., 2014; Hogan, Mata, \& Carstensen, 2013; Lin et al., 2013; Loy, O’Connor, \& Dishman, 2013). Lifelong exercise participation is influenced by exercise history, in that an active young adult can continue exercising as an older adult (Chatfield, 2015). Although young and mid-age adults (18-54 years old) report the highest exercise levels $(56.4 \%$ for $18-44$ year olds and $47.9 \%$ for $45-54$ year olds), there still remain noteworthy disparities in meeting the minimum exercise guidelines - 150 minutes/week of moderate-to-vigorous aerobic exercise - based on educational attainment, race/ethnicity, and income (U.S. Department of Health \& Human Services [USDHHS], 2017). Specifically, only $29.8 \%$ of non-high-school completers report being regularly active compared with $38.7 \%$ of high-school completes and $60.5 \%$ of college graduates. Additionally, $41.8 \%$ of African Americans report exercising regularly compared with 50.9\% of Whites. Similarly, only $35.4 \%$ of those whose family incomes were $<100 \%$ of the lowest poverty threshold report meeting the minimum exercise recommendations compared with $64.7 \%$ of those whose family incomes were $600+\%$ of the poverty threshold (USDHHS, 2017).

Although there is an abundance of behavior-change interventions to increase exercise levels, the aforementioned findings - noted self-reported exercise levels tend to be over- 
estimated - are discouraging, indicating that there might be a widening disconnect between the needs of the public and the content of the exercise promotion interventions (Kosma \& Buchanan, 2018; Kosma, Buchanan, \& Hondzinski, 2015). Instead of relying on pre-identified, cut-and-dried theory-based determinants of exercise behavior - e.g., positive exercise attitudes and self-efficacy should lead to increased exercise levels - a more practical (phronetic) approach has been proposed, whereby exercise participation can be influenced by people's value systems (Kosma \& Buchanan, 2018; Kosma et al., 2015). The meaning and importance of exercise values can be instilled when movement and exercise are part of history, society, and culture. People find meaning in activities that they hold dear; activities that are consciously and sub-consciously learned within one's culture, such as healthy diet and exercise (Bourdieu, 1994; Sayer, 2011; Wolf, 2015). In this way, wise (phronetic) decisions about making exercise part of life are also enhanced (Angier, 2010; Aristotle, 350 B.C.E/1962; Kosma \& Buchanan, 2018; Kosma, Hondzinski, \& Buchanan, 2017). On the contrary, jumping on the bandwagon that global neoliberal capitalism - e.g., purchasing the latest technological gadgets to be active - should be the cultural norm of the $21^{\text {st }}$ century has been heavily criticized for promoting sick-care - rather than health-care - societies where autonomy and wise (phronetic) actions about healthy lifestyles are jeopardized (Buchanan, 2016; Kosma \& Buchanan, 2018; Marvasti, \& Stafford, 2012).

The major challenge of examining values (e.g., reasons for exercising or not) is that they cannot be empirically measured because they cannot be seen or touched or smelled (Buchanan, 2016; Flyvbjerg, 2001, 2004; Kosma et al., 2015). However, values can be evaluated and understood by reinforcing a democratic and in-depth dialogue between the researcher and the community members regarding their needs and goals (Buchanan, 2004; Kosma et al., 2015). Therefore, phonetic research is community-oriented where the importance and content of exercise promotion programs derive from an open dialogue between the community members (co-investigators) and the researchers. Such research is mainly qualitative in nature - though mixed-methods designs are also used (Kosma et al., 2017), with the goal being to shed light on the meaning of exercise.

Based on a recent systematic review, most research on exercise facilitators and barriers has been conducted among midlife White adults (Kelly et al., 2016). In another systematic review of qualitative studies regarding exercise impediments and enablers among African Americans, it was reported that most studies were conducted among women and middle-aged adults (Siddiqi, Tiro, \& Shuval, 2011). The review results in the latter study were organized around the socio-ecological framework emphasizing individual exercise barriers (e.g., lack of time, motivation) and social and environmental exercise barriers (e.g., lack of childcare, family responsibilities, safety issues). Some of the reported exercise enablers were health benefits, enjoyment, weight loss, and body shape maintenance. Exercise behavior was not measured and several qualitative studies ascribe to the logical positivism paradigm, relying on certain theoretical frame- works with hypothesized exercise predictors (e.g., self-efficacy and intention); therefore, it is uncertain if people's responses are biased and predetermined or if they reflect actual exercise values within a certain context, time, and socio-political system. On the contrary, in phronetic research people's values regarding lifestyle behaviors (e.g., exercise) are viewed as qualitatively distinct and their existence and meaning can widely vary (Buchanan, 2000; Kosma et al., 2015). Therefore, the purpose of this phronetic, qualitative study was to examine the exercise behavior and qualitatively distinct exercise values (e.g., activity and inactivity reasons) within one's unique exercise context among educationally and economically disadvantaged African American young adults.

\section{METHODS}

\section{Design and Procedures}

Drawing on phronetic research (Flyvbjerg, 2001), this was a qualitative study among 14 African American young adults, who attended classes for their General Educational Development (GED) exam in an inner-city learning center. The GED center director and the first study author recruited the participants based on their diverse exercise experiences and levels. All young adults who expressed interest in the project were included in the study. One individual was excluded from the study because she was an older adult (68 years old). Personal interviews, lasting between 19 - 39 minutes, were conducted by the first author in a quiet room at the learning center. Prior to the interviews, Institutional Review Board approval was obtained and participants signed the study's consent form. These data reflect the first study of a larger research project with the goal being to examine the relations among depression, exercise behavior, screen-viewing time, and educational challenges for the study sample. Interview questions reflected on participants' exercise behavior (e.g., type, frequency, intensity, and duration), positive and negative exercise experiences, exercise reasons or not, exercise of peers, and neighborhood safety (see Table 1). The interview process was dialogical in nature to build trust and engage in in-depth discussions regarding exercise behavior and values. Participants were also asked about their age, gender, ethnicity, education, income, and number of household members. Based on discussions with the GED center director, the first author initially drafted the interview guide, which was then reviewed by the second author for revisions and recommendations. The final questions were then pilot-tested with two participants, whose results were included in the study.

\section{Data Analysis}

Audiotapes of the interviews were transcribed verbatim and the interviewer double-checked transcription accuracy. Pseudonyms were used to report study results. The development of themes and sub-themes was informed by the analytical steps of phronetic research (Braun \& Clarke, 2006; Flyvbjerg, 2001, 2004; Kaffle, 2011): a) after entering the transcripts and interview notes in NVivo 10 the first author 
repeatedly read and reread the data to become familiar with the participants' stories; b) the first author coded the data in a systematic fashion and developed themes and sub-themes based on each individual story and the entire data set - a process also known as the hermeneutic circle (Kafle, 2011); c) the second study author independently reviewed the coded data and the two authors finalized the themes and sub-themes via consensus discussion; d) compelling extracts (quotes) were selected relevant to the coded data, the entire data set, research questions, and literature. Key indicators in testing the rigor of this phronetic research involved the emphasis on values, the involvement of participants and the GED center director in developing the interview guide, the dialogical nature of the interviews, and the in-depth examination and report of the participants' stories (Flyvbjerg, 2001, 2004; Kafle, 2011; Kosma et al., 2015).

\section{RESULTS}

\section{Participant Characteristics}

The average age of the participants was 32.97 years old $( \pm 14.13$; age range: $18-57)$. Ten out of the 14 participants were young adults (age range: $18-43$ ), while four participants were middle-aged: 46-57 years old. The study included nine women and five men. Of the 14 participants, 13 were African American and one was a White female. Most of the people reporting gross annual household incomes $(n=9)$ were at poverty level (from $\$ 1,680$ to $\$ 17,000$ ) (U.S. Census Bureau, 2017), while there was only one individual with higher income (\$128,000/year). Only one participant reported living alone, while the others lived with parents and/or siblings adding to their financial difficulties. The highest school grade attained varied from $8^{\text {th }}$ grade to $12^{\text {th }}$ grade. Participant characteristics are outlined in Table 2.

\section{Exercise Behavior}

Participants' reported exercise levels are delineated in Table 3. Three men met the aerobic exercise recommendations and their main activity was basketball. Three individuals were somewhat active, while the rest of the participants were inactive. Although three of the inactive participants walked for transportation, none of them incorporated walking into an exercise routine. Several participants reported performing some strengthening activities, mainly push-ups and sit-ups.

\section{Emerging Themes and Sub-themes}

The two themes of the phronetic analysis included exercise facilitators (e.g., enjoyment, health improvement, and weight loss - toned physique) and exercise barriers (e.g. time constraints and other priorities, injuries, and accessibility and safety issues).

\section{Theme 1: Exercise Facilitators}

The first theme emerged from responses on exercise reasons and positive exercise experiences. In a descending order
Table 1. Interview guide

Are you physically active? In what types of exercises do you currently participate?

How many days per week do you exercise?

For how long do you exercise each time?

How hard do you exercise?

If not active now, why aren't you active?

What does exercise mean to you? What comes to mind when you hear the term "exercise"?

Describe a positive exercise experience. Why was this experience positive?

Describe a negative exercise experience if you had one. Why was this experience negative?

Can you describe similar or different exercise experiences of your peers?

Are your peers and others (neighbors, friends, family) active?

If you see people exercising, what do you think?

Is it safe to exercise in your neighborhood?

Table 2. Demographic profile

\begin{tabular}{lcc}
\hline Variable & $\mathbf{N}$ & $\mathbf{\%}$ \\
\hline Gender & 9 & \\
Female & 5 & 64.29 \\
$\quad$ Male & & 35.71 \\
Race & 13 & 92.86 \\
$\quad$ African American & 1 & 7 \\
$\quad$ Caucasian & & \\
Education & 6 & 42.86 \\
$8^{\text {th }}$ to $10^{\text {th }}$ grade & 6 & 42.86 \\
$11^{\text {th }}$ grade & 2 & 14.29 \\
$\quad 12^{\text {th }}$ grade - no degree & & \\
Gross annual income $(n=9)$ & 3 & 21.43 \\
$<\$ 12,000$ & 3 & 21.43 \\
$\$ 12,000-\$ 17,160$ & 3 & 21.43 \\
$\$ 42,000-\$ 128,000$ & 5 & 35.71 \\
$\quad$ Missing &
\end{tabular}

of frequency report, the main subthemes were enjoyment ( $n=13)$, health improvement $(n=10)$, weight loss and toned physique $(n=8)$, and utilitarian purpose $(n=1)$.

\section{Enjoyment}

Several participants mentioned that they loved exercising because it "builds up stamina", "strengthens muscles", and "gets one in shape." Two of the three active men enjoyed playing basketball because it was part of their lives. Baylor enjoyed tennis because it was "almost second nature" and "required patience, you know, hand-eye coordination." Demingo said that basketball was his "comfort area." For Baylor,

"ball is life... you can compare basketball to life. gotta know when to move, where to go to, how to shoot 
Table 3. Exercise levels based on aerobic recommendations

\begin{tabular}{|c|c|c|c|c|}
\hline Name & Type & Frequency & Duration & Intensity \\
\hline \multicolumn{5}{|l|}{ Active } \\
\hline Baylor & $\begin{array}{l}\text { Basketball } \\
25-30 \text { push ups }\end{array}$ & $\begin{array}{l}\text { 3-4 days/week } \\
\text { 2-3 days/month }\end{array}$ & 2 hours/day & Hard \\
\hline Demingo & $\begin{array}{l}\text { Basketball } \\
\text { Rides bike to go to GED center } \\
20 \text { push ups }\end{array}$ & $\begin{array}{l}5 \text { days/week } \\
4 \text { days/week }\end{array}$ & $\begin{array}{l}3 \text { hours/day } \\
3 \text { miles/day }\end{array}$ & $\begin{array}{l}\text { Runs full court } \\
\text { Comfortably }\end{array}$ \\
\hline Hunner & $\begin{array}{l}\text { Basketball } \\
\text { Runs }\end{array}$ & $\begin{array}{l}2-3 \text { days/week } \\
1 \text { day/week }\end{array}$ & $\begin{array}{l}3 \text { hours/day } \\
1 \text { hour }\end{array}$ & $\begin{array}{l}\text { Moderate } \\
\text { Moderate }\end{array}$ \\
\hline \multicolumn{5}{|c|}{ Somewhat active } \\
\hline Donoban & $\begin{array}{l}\text { Runs } \\
200 \text { sit ups } \\
60 \text { push-ups }\end{array}$ & $\begin{array}{l}4 \text { days/week } \\
\text { Daily } \\
\text { Daily }\end{array}$ & $\begin{array}{l}12 \text { minutes } \\
20 \text { minutes } \\
10-15 \text { minutes }\end{array}$ & Medium speed \\
\hline Lasonne & Walking for exercise & 2 days/week & 45 minutes/day & Comfortably \\
\hline Tilika & $\begin{array}{l}\text { Aerobics at gym (walking, arm and leg } \\
\text { pedaling) }\end{array}$ & 2 days/week & 24 minutes/day & Comfortably \\
\hline \multicolumn{5}{|l|}{ Inactive } \\
\hline Darlene & None & & & \\
\hline Jerrica & Walking for transportation & 2 days/week & 3 miles $/$ time & \\
\hline Kinley & 60 push-ups and 60 sit-ups & Every night & & \\
\hline Lanaia & None & & & \\
\hline Rachel & $\begin{array}{l}\text { Push-ups } \\
\text { Walking for transportation }\end{array}$ & $\begin{array}{l}\text { Twice/day } \\
4 \text { days/week }\end{array}$ & $\begin{array}{l}5-6 / \text { time } \\
1 \text { hour/time }\end{array}$ & $\begin{array}{l}\text { Leisurely, talking } \\
\text { and walking }\end{array}$ \\
\hline Sondra & Walking for transportation & & & \\
\hline Trevor & Push-ups, sit-ups, stretching & Twice/week & 30 minutes & 10 push-ups/minute \\
\hline Trinity & None & & & \\
\hline
\end{tabular}

the ball, how hard to shoot the ball, the angle to shoot at, you gotta be on point when you play basketball... cause the ball not gonna go in if you don't use perfect form. Same way with life. If you don't use the right, you know, form in life, you won't get nowhere."

Four out of eight participants referred to past enjoyable exercise experiences like dancing and swimming. Darlene recalled how she loved dancing and being a cheerleader in high school. Although she does not currently dance, she and her kids "will have music in the house and play around." For Trinity, water exercise at the YMCA "felt good and relaxing." Jerrica loved swimming, especially following her back injury because "the water relieves some of that tension and pressure off my back so that I can loosen up enough to really move around."

\section{Health improvement}

Although most participants generally referred to the positive effects of exercise on health, a few individuals expressed specific positive exercise effects, such as decreasing cholesterol and sugar levels, preventing heart attacks, improving cognitive function, and increasing energy levels. Although Lasonne does not meet exercise recommendations (see Table 3 ), she is satisfied with her exercise level because "walking... is good for your heart. It causes less heart attacks and blockage in the heart." Tilika has Crohn's disease and exercise to her means improved functioning:
"...I could see myself. My legs were getting too tired and I was getting too heavy in my legs and the doctor was telling me... I need to get some exercise 'cause of my condition. ...for my health... keeping my sugar down and my cholesterol is not that high like it was. And my body weight is down. And... I'm not that tired like I used to be."

A few participants reinforced the psychological benefits of exercise, such as "stress relief." Rachel said: “...get in shape, bear up your body, it make you feel good." Trinity emphasized both the positive psychological and cognitive effects of exercise:

"Just being in good health, feeling good, thinking real good... I have experienced that... when you take away from that kind of diet (heavy starches) and exercise, you more... focused. energetic."

\section{Weight loss and toned physique}

Several participants, including two of the three active men, stated that exercise helped them lose weight and develop toned muscles. Demingo mentioned that constant exercise can cause bodily changes like "thinner face." Lanaia feels that cardio and weight training would help with "toning, shaping up, and getting more fit." Huner said that basketball helped him "lose a lot of pounds." He believes that exercise will help him as follows: 
"Exercise means trying to get your life back together... get your body right. My arms are very toned, but it's like my stomach is not right. I need to start going back to the gym... sometimes I need help with my calf muscles... they're kinda flabby."

\section{Utilitarian purpose}

Although Jerrica loved both her past Karate and swimming exercise experiences, she would pick Karate because during college she would be working for campus security:

“...I'm looking to eventually become a Poetry Therapist... I still work for the church on Sundays as a Security Guard... So karate would definitely probably be my number one pick because while I'm in college... I'm gonna be working for Campus Security."

\section{Theme 2: Exercise Barriers}

The second theme emerged from questions related to inactivity reasons, negative exercise experiences, and neighborhood safety. In a descending order of frequency report, common exercise barriers included time constraints and other priorities $(n=7)$, injuries $(n=5)$, accessibility and cost issues $(n=4)$, safety issues $(n=4)$, personality $(n=3)$, and undesirable results on appearance and low performance $(n=3)$.

\section{Time constraints and other priorities}

Inactive participants and all three insufficiently active people reported that work, school, family, and caring responsibilities prevented them from starting an exercise program or participating in regular exercise. Lasonne said that she cares for her grandson during the day until his parents come home. Darlene - a single mom four days per week - mentioned how caring for her four children and tending to school responsibilities make it impossible for her to exercise:

"My day look like getting up in the morning, um, getting my kids together for school... and now since I have started school I have to get myself together and... come here and learn. And once I leave here I have to go home and cook a meal, fix my kids something to eat, help with homework, wash clothes, get their bed clothes together to start the next day."

\section{Injuries}

Five participants reported that such injuries as fractured bones, pulled muscles, back injuries, and leg crumps resulted in discontinuance of exercise participation. Baylor had to stop his favorite sport, football, because of "bruised ribs and fractured bones." Jerrica hurt her back and foot, thus exercising becomes challenging:

"I have a back injury from when I was a teenager and it's really hard and difficult for me to do certain exercises. I'm not supposed to do any extension exercises. I walked and I hurt my foot. It healed wrong and, um, now I have bone spurs in my foot...I've pulled muscles in my back before lifting weights... other than that, I like exercising."

\section{Accessibility and cost issues}

Four participants commented on accessibility and financial issues that keep them from joining a gym membership. Although Jerrica loves swimming, she does not have access to a pool. Rachel mentioned that she preferred structured exercise classes where an expert could show her how to exercise, but she "did not have the money to pay for membership." Lanaia also preferred going to the gym and using the machines because "it feels more relaxing and they work different places", but financial reasons prevented her from joining a gym.

\section{Safety issues}

Although only one woman mentioned that safety issues in her neighborhood prevented her from walking alone, three other women stated that their neighborhood was unsafe to walk. Jerrica said that she lives in a "rough area" and "there's not a lot of people who walk or jog or ride bikes." Tilika said that her apartment is "on a highway and behind it they are selling drugs." Trinity highlighted that when her friend stopped walking with her she also stopped because it is dangerous to walk alone in her neighborhood in the evenings.

\section{Personality}

Three individuals mentioned that their personality was the main reason for not exercising. For example, Sondra is "stubborn" to change her habits. Trevor said that he is "lazy" and simply "does not want to do it." Trinity needs to be disciplined and manage her time "a bit more wisely."

\section{Undesirable results on appearance and low performance}

Three individuals linked negative exercise experiences and poor diet to undesirable physique changes and low performance. Hunner and Lanaia mentioned that poor diet (fast food and lots of starch) led to weight gain and low energy and stamina during exercise. Hunner was really concerned about his weight gain due to unhealthy diet, and thus "loss of stamina."

Lanaia is discouraged from exercising because she does not see fast and desirable results, especially when her diet is poor:

“...I get discouraged a lot. I don't see results quick enough. I want to be firmer, tighter, you know, lean more. I used to be a vegetarian and I used to work out daily... I went on a starch diet and it ruined my body... It made me really lazy and drowsy, so I wasn't really in the mood... I do see small results, but they're not like, oh my gosh results."

\section{DISCUSSION}

The purpose of this phronetic, qualitative study was to examine the exercise behavior and values of socio-economically disadvantaged African American young adults at an inner-city learning center. Two themes, exercise facilitators and barriers, and several sub-themes emerged from the analysis. The most commonly reported exercise facilitators 
were enjoyment, health improvement, weight loss and toned physique, and utilitarian purpose. Although weight loss and physique maintenance are common exercise facilitators among African American young adults (Bragg, Tucker, Kaye, \& Desmond, 2009), health benefits are typically cited by middle-aged adults and older adults (Kirchhoff, Elliott, Schlichting, \& Chin, 2008; Kosma et al., 2017, respective1y). Several of the participants in this study had health issues, especially mental problems (e.g., depression) for the young adults, thus the potential reason for valuing the health benefits of exercise. Although enjoyment is typically cited as an exercise facilitator among African American women (Bopp et al., 2007), a unique aspect in this study is that the reasons for activity enjoyment - especially among the active African American men - included increasing fitness and skill development and viewing exercise as part of their lives. This supports the concept of phronesis, in that the value and joy of exercise stem from its inherent qualities during practice like increased fitness and skill level (Kosma \& Buchanan, 2018). Baylor's confidence for tennis increased when his skill and technique developed. Baylor also loves basketball - his comfort area - because he learned the "proper form/ technique." Another unique result reflects the utilitarian pick of exercise type (Karate) for Jerrica because of her work for campus security.

Important exercise barriers included time constraints and other priorities, injuries, accessibility and cost issues, safety issues, personality, and undesirable results on appearance and low performance. Time constraints is a commonly reported exercise barrier among young adults (Bopp et al., 2007) who have employment and/or school responsibilities. Although injuries are commonly reported exercise barriers (Siddiqi et al., 2011), some of the study's active and somewhat active participants managed to overcome their injuries and return to exercise. The ability to overcome the negative effects of injury, allow time to recover, and return to a regular exercise lifestyle has also been reported among older African American women (Kosma et al., 2017). Accessibility difficulties, cost issues, and safety concerns are typical exercise barriers among African Americans, especially of lower SES (Kosma et al., 2017). Safety concerns was a barrier reported by only women, highlighting the importance to identify safe exercise settings for them.

Surprisingly, personality (e.g., lack of motivation and discipline) did not come up as a frequent exercise barrier, even though individualistic approaches dominate in exercise promotion. The three regularly active participants - all men - were mainly playing basketball with their friends and other family members. It seems that this playful, group-based activity kept these young men regularly active. Exercise classes and social support are important exercise motivators among African American men and women (Siddiqi et al., 2011). Basketball is a popular sport in the African American community, especially among men (Sailes, 2017). Dancing is another popular activity in the African American culture, especially among women (Maultsby, 2000). Darlene - an inactive participant - recalls how dancing with her kids gives her great pleasure - noted that she loves dancing and she used to be a cheerleader at high school. Taken together, these results indicate that playful activities should be emphasized not only among children, but also for all age groups (Kosma et al., 2017). Culturally meaningful activities can best instill the value of movement because, contrary to the dominant paradigm, movement is not an individualistic experience but a shared one with the surrounding world (Kosma \& Buchanan, 2018; Kosma et al., 2017; Merleau-Ponty, 1945/2002).

Another unique finding was how undesirable exercise effects on appearance and performance linked to poor diet and hindered exercise participation. Both Hunner (active participant) and Lanaia (inactive participant) connected poor diet with decreased exercise performance and undesirable body physique. Although attempting to maintain popular and at times unrealistic western body ideals (e.g., thin, strong, and full-breasted female bodies) can hinder exercise participation (Segar, Spruijt-Metz, \& Nolen-Hoeksema, 2006), improving body shape and maintaining a healthy weight by becoming fit - as part of one's happiness and not necessarily a way to show off - can be an important exercise motivator (Coffey, 2015). Some of the somewhat active (Donoban) and aerobically inactive (Rachel) participants performed strengthening activities to maintain a healthy weight and be in shape.

Although most people were knowledgeable of the exercise benefits, only three people were regularly active. Knowledge alone about the importance of exercise does not necessarily translate into action; exercise needs to be playful and culturally meaningful to be experienced and valued (Kosma \& Buchanan, 2018; Kosma et al., 2017). All three regularly active participants were men and men tend to be more active than women (USDHHS, 2017). Only women did emphasize childcare responsibilities as exercise barriers. Therefore, a switch in the cultural norm is necessary, where men should assume increasing childcare responsibilities so that women can have more free time to exercise. Such cultural switch can start via policy change where long-term and fully-paid maternity and paternal leave is instituted with flexible employment schedules for especially young mothers. Following the comparison of 21 high-income countries, including USA, Ray, Gornick, and Schmitt (2010) found that the countries with the highest generosity and gender equality in parental leave were Finland, Norway, Sweden, and Greece. In such a policy model, women can rejoice motherhood without being forced and/or manipulated to be unemployed and/or be preoccupied with only household chores without leisure activities, such as exercising. Although result generalizability is limited to similar populations, this study has numerous strengths. It took place in a disadvantaged community among African Americans of lower SES. It included both women and men of younger age compared with the majority of studies that report on middle-aged White women. Contrary to many qualitative studies that follow the logical positivism paradigm, a phronetic approach was used here to delineate situation and time-specific exercise behavior and values.

Some study implications for exercise promoters and researchers are as follows: a) emphasizing playful, culturally meaningful, and socially supported activities for fitness and 
skill development in order to enhance exercise participation and enjoyment; b) instead of only empowering women, involving also men in discussions regarding their role on childcare responsibilities; c) reinforcing policy change via the provision of long-term and fully-paid maternity and paternal leave; d) identifying safe and accessible exercise settings, especially among women of lower SES; e) enhancing knowledge about safe exercises and smooth recovery from injury to respectively decrease the risk of injuries and strengthen the return to exercise participation; and f) emphasizing fit and healthy physiques for personal fulfilment and enjoyment.

\section{CONCLUSION}

Contrary to the logical positivism paradigm, in this phronetic, qualitative study exercise values were delineated in relation to unique exercise experiences within one's world among socio-economically disadvantaged African American young men and women. Based on the participants' qualitatively distinct exercise experiences, the value of exercise can be best instilled if it is linked to playful and culturally meaningful exercises where the emphasis is on the inherent qualities of movement, such as skill, fitness, and physique improvement within a socially supported setting. Policy change - e.g., provision of long-term and fully paid maternity and paternal leave and safe exercise settings - is essential for enhanced exercise participation, especially among women of lower SES. Information about injury prevention and recovery is also essential to respectively avoid exercise interruptions and enhance the return to meaningful exercise participation.

\section{REFERENCES}

Angier, T. (2010). Techné in Aristotle's ethics: Crafting the moral life. New York, NY: Continuum International.

Aristotle. (1962). Nichomachean ethics (M. Ostwald, Trans.). Indianapolis, IN: Bobbs-Merrill (Original work published 350 B.C.E).

Bopp, M., Lattimore, D., Wilcox, S., Laken, M., McClorin, L., Swinton, R.,... Bryant, D. (2007). Understanding physical activity participation in members of an African American church: A qualitative study. Health Education Research, 22, 815-826. https://doi.org/10.1093/her/ cyl149

Bourdieu, P. (1994). Practical reason. Cambridge, UK: Polity.

Bragg, M. A., Tucker, C. M., Kaye, L. B., \& Desmond, F. (2009). Motivators of and barriers to engaging in physical activity: Perspectives of low-income culturally diverse adolescents and adults. American Journal of Health Education, 40, 146-154.

Braun, V., \& Clarke, V. (2006). Using thematic analysis in psychology. Qualitative Research in Psychology, 3, 77101. doi:10:1191/1478088706qp063oa

Buchanan, D. R. (2000). An ethic for health promotion: Rethinking the sources of human well-being. New York, NY: Oxford University Press.

Buchanan, D. R. (2016). Promoting dignity: The ethical dimension of health. International Quarter- ly of Community Health Education, 36, 99-104. doi:10.1177/0272684X16630885

Buchanan, D. R. (2004). Two models for defining the relationship between theory and practice in nutrition education: Is the scientific method meeting our needs? Journal of Nutrition Education and Behavior, 36, 146-154. doi:10.1016/S1499-4046(06)60152-8

Chatfield, S. L. (2015). Characteristics of lifelong physically active older adults. Qualitative Health Research, 25, 966-973. doi:10.1177/1049732314554095

Coffey, J. (2015). 'As long as I'm fit and a healthy weight, I don't feel bad': Exploring body work and health through the concept of 'affect'. Journal of Sociology, 51, 613627. doi:10.1177/1440783313518249

Flyvbjerg, B. (2001). Making social science matter: Why social inquiry fails and how it can succeed again. Cambridge, England: Cambridge University Press.

Flyvbjerg, B. (2004). Phronetic planning research: Theoretical and methodological reflections. Planning Theory \& Practice, 5, 283-306. doi:10.1080/1464935042000250195

Gerber, M., Branda, S., Herrmann, C., Colledge, F., Holsboer-Trachsler, E., \& Pühse, U. (2014). Increased objectively assessed vigorous-intensity exercise is associated with reduced stress, increased mental health and good objective and subjective sleep in young adults. Physiology \& Behavior, 135, 17-24. http://dx.doi.org/10.1016/j. physbeh.2014.05.047

Hogan, C. L., Mata, J., \& Carstensen, L. L. (2013). Exercise holds immediate benefits for affect and cognition in younger and older adults. Psychology and Aging, 28, 587-594. doi:10.1037/a0032634

Kafle, N. P. (2011). Hermeneutic phenomenological research method simplified. Bodhi: An Interdisciplinary Journal, 5, 181-200. http://dx.doi.org/10.3126/bodhi.v5i1.8053

Kelly, S., Martin, S., Kuhn, I., Cowan, A., Brayne, C., \& Lafortune, L. (2016) Barriers and facilitators to the uptake and maintenance of healthy behaviours by people at mid-life: A rapid systematic review. PLOS ONE, 11, e0145074. doi:10.1371/journal.pone.0145074

Kirchhoff, A. C., Elliott, L., Schlichting, J. A., \& Chin, M. H. (2008). Strategies for physical activity maintenance in African American women. American Journal of Health Behavior, 32, 517-524.

Kosma, M., \& Buchanan, D. R. (2018). "Connect,” log it, track it, go! Techne - not technology — and embodiment to achieve phronesis in exercise promotion, Quest, 70, 100-113. doi:10.1080/00336297.2017.1355818.

Kosma, M., Hondzinski, J., \& Buchanan, D. R. (2017). Exercise, health, and falls risks among older African American women. International Journal of Kinesiology \& Sports Science, 5, 16-27. http://dx.doi.org/10.7575/aiac. ijkss.v.5n.3p. 16

Kosma, M., Buchanan, D. R., \& Hondzinski, J. (2015). The role of values in promoting physical activity. Quest, 67, 241-254. doi:10.1080/00336297.2015.1050117

Lin, X., Zhang, X., Guo, J., Roberts, C. K., McKenzie, S., Wu, W-C.,... Song, Y. (2015). Effects of exercise training on cardiorespiratory fitness and biomarkers of cardiometa- 
bolic health: A systematic review and meta-analysis of randomized controlled trials. Journal of the American Heart Association, 4, e002014. doi:10.1161/JAHA.115.002014

Loy, B. D., O’Connor, P. J., \& Dishman, R. K. (2013). The effect of a single bout of exercise on energy and fatigue states: A systematic review and meta-analysis. Fatigue: Biomedicine, Health \& Behavior, 1, 223-242.

Marvasti, F. F., \& Stafford, R. S. (2012). From sick care to health care - Reengineering prevention into the U.S system. New England Journal of Medicine, 367, 889891. doi:10.1056/NEJMp1206230

Maultsby, P. K. (2000). Africanisms in African American music. In F. W. Hayes (Ed.), A turbulent voyage: Readings in African American studies ( $3^{\text {rd }}$ ed.) (pp. 156-176). Lanham, MD: Rowman \& Littlefield Publishers, Inc.

Merleau-Ponty, M. (2002). Phenomenology of perception (C. Smith, Trans.). London, England: Routledge (Original work published 1945).

Ray, R., Gornick, J. C., \& Schmitt, J. (2010). Who cares? Assessing generosity and gender equality in parental leave policy designs in 21 countries. Journal of European Social Policy, 20, 196-216. https://doi. org/10.1177/0958928710364434

Sailes, G. A. (Ed.). (2017). African Americans in sport: Contemporary themes. New York, NY: Routlege.
Sayer, A. (2011). Why things matter to people: Social science, values and ethical life. Cambridge, UK: Cambridge University Press.

Segar, M., Spruijt-Metz, D., \& Nolen-Hoeksema, S. (2006). Go figure? Body-shape motives are associated with decreased physical activity participation among midlife women. Sex Roles, 54, 175-187. doi:10.1007/s11199006-9336-5

Siddiqi, Z., Tiro, J. A., \& Shuval, K. (2011). Understanding impediments and enablers to physical activity among African American adults: A systematic review of qualitative studies. Health Education Research, 26, 1010-1024. doi:10.1093/her/cyr068

United States Census Bureau. (2017). Poverty. Retrieved from https://www.census.gov/topics/income-poverty/ poverty/about.html

United States Department of Health and Human Services (USDHHS), Office of Disease Prevention and Health Promotion. (2017). Healthy people, 2020, topics and objectives-physical activity. Retrieved from http:// www.healthypeople.gov/2020/topics-objectives/topic/ physical-activity

Wolf, S. (2015). The variety of values: Essays on morality, meaning, \& love. New York, NY: Oxford University Press. 